\title{
Review of: "Lower Geriatric Nutritional Risk Index Are Associated With A Higher Incidence Of Osteoporosis In Northern China Type 2 Diabetes"
}

bolin $\mathrm{li}^{1}$

1 Xi'an Jiaotong University

Potential competing interests: The author(s) declared that no potential competing interests exist.

I have read the article by Yuanyuan Ji et al entitled " Lower Geriatric Nutritional Risk Index are associated with a higher incidence of osteoporosis in northern China type 2 diabetes ". In this article, the authors emphasized that lower GNRI among type 2 diabetic patients in northern China are associated with a higher Incidence of osteoporosis. However, there are a number of specific questions.

Major points

1) Please provides a theoretical basis for evaluating and choosing the ways of grouping.

2) Please perform logistic regression analysis to assess the relationship between GNRI and osteoporosis in the whole cohort population.

3) Some significant baseline variables were not adjusted in Multivariate analyses. What is the reason?

Minor points

1) Some sentences are not well expressed. For example, "the prevalence of diabetic osteoporosis accounts for approximately more than $50 \%$ of diabetic patients" in the first paragraph of introduction. 